\title{
X-RAY FLARES AND MASS OUTFLOWS DRIVEN BY MAGNETIC INTERACTION BETWEEN A PROTOSTAR AND ITS SURROUNDING DISK
}

\author{
M. R. HAYASHI ${ }^{1}$, K. SHIBATA ${ }^{2}$,
}

and

R. MATSUMOTO 3,4

Received __; accepted _

\footnotetext{
${ }^{1}$ Department of Physics, Graduate School of Science and Technology, Chiba University, Inage-ku, Chiba 263, Japan ; hayashi@spica.c.chiba-u.ac.jp

${ }^{2}$ National Astronomical Observatory, Mitaka, Tokyo 181, Japan

${ }^{3}$ Department of Physics, Faculty of Science, Chiba University, Inage-ku, Chiba 263, Japan

${ }^{4}$ Advanced Science Research Center, JAERI, Naka, Japan
} 


\begin{abstract}
We propose a model of hard X-ray flares in protostars observed by ASCA satellite. Assuming that the dipole magnetic field of the protostar threads the protostellar disk, we carried out 2.5-dimensional magnetohydrodynamic (MHD) simulations of the disk-star interaction. The closed magnetic loops connecting the central star and the disk are twisted by the rotation of the disk. As the twist accumulates, magnetic loops expand and finally approach to the open field configuration. A current sheet is formed inside the expanding loops. In the presence of resistivity, magnetic reconnection takes place in the current sheet. Outgoing magnetic island and post flare loops are formed as a result of the reconnection. The time scale of this 'flare' is the order of the rotation period of the disk. The released magnetic energy partly goes into the thermal energy and heats up the flaring plasma up to $10^{8} \mathrm{~K}$. The length of the flaring loop is several times of the radius of the central star, consistent with observations. The speed of the hot plasmoid ejected by the reconnection is $200-400 \mathrm{~km} \mathrm{~s}^{-1}$ when the footpoint of the loop is at $0.03 \mathrm{AU}$ from $1 \mathrm{M}_{\odot}$ protostar. The hot plasma outflow can explain the speed and mass flow rate of optical jets. Dense, cold, magnetically accelerated wind $\left(v \sim 150-250 \mathrm{~km} \mathrm{~s}^{-1}\right)$ emanates from the surface of the disk along the partially open magnetic field lines threading the disk. This dense, cold wind may correspond to high velocity neutral winds.
\end{abstract}

Subject headings: stars: activity — stars: pre-main sequence — ISM: jets and outflows - accretion, accretion disks - MHD 


\section{INTRODUCTION}

Observations by ASCA revealed that protostars are strong hard X-ray emitting sources. By using ASCA, Koyama et al. (1994,1996a,b) carried out a systematic survey of hard X-ray sources in molecular clouds. They detected hard X-rays (4-10keV) associated with Class I IR sources (protostars). A protostar (R1 in Coronet cluster) exhibited a powerful X-ray flare whose spectrum showed significantly higher temperature $\left(T \sim 10^{8} \mathrm{~K}\right)$ than X-ray flares in $\mathrm{T}$ Tauri stars which is usually $\sim(1-2) \times 10^{7} \mathrm{~K}$ (Koyama et al. 1996b). The X-ray luminosity in the $2-10 \mathrm{keV}$ band is $\sim 10^{31} \mathrm{erg} \mathrm{s}^{-1}$ and the e-folding time of the flare is $\sim 5 \times 10^{4} \mathrm{sec}$ (Koyama et al. 1996b). Three X-ray emitting class I sources in Rho Oph cloud also showed significantly hard X-rays. One of them showed flare like time variabilities (Koyama et al. 1994).

Although the analogy with solar flares may be appropriate for such X-ray flares in protostars, they differ from solar flares in the following points: (1) The total energy released by the protostellar flare $\left(10^{35}-10^{36} \mathrm{erg}\right)$ is $10^{5}$ times larger than solar flares. (2) The

spectrum of the X-ray emission in protostellar flares is harder than solar flares, indicating the existence of $8 \mathrm{keV}$ plasma, and (3) The size of the X-ray emitting region is the order of $10^{11} \mathrm{~cm}$ (Koyama 1995 private communication). If the emission comes from magnetic loops, their size should be several times larger than the protostar.

It has long been recognized that solar flares are often associated with sheared magnetic fields. Mikić \& Linker (1994) and Linker \& Mikić (1995) carried out MHD simulations of the dynamical evolution of magnetic arcades in spherical geometry under shearing motions imposed on the line-tied footpoints of an initially current free arcade. When the amount of the footpoint shear is sufficiently large, the magnetic arcades begin to inflate. In the presence of finite resistivity, magnetic nonequilibrium leads to the magnetic reconnection and plasmoid ejection in the current sheet developed inside the expanding arcades. 
In the innermost region of protostellar disks, the magnetic fields of the protostar will affect the dynamical evolution of the disk (e.g., Camenzind 1990). Since the rotation of the disk continuously injects helicity into the closed magnetic fields connecting the central star and the disk, we expect magnetic nonequilibrium and resulting magnetic reconnection similar to those in solar flares (see also Ghosh \& Lamb 1979, 1991; Zylstra 1988; Lynden-Bell \& Boily 1994). Recently, the magnetic interaction between a central star and its surrounding disk has attracted a renewed interest in relation to the activity of young stars (Shu et al. 1994, Hirose 1994, Lovelace et al. 1995). In order to explain why T Tauri stars have rotation rates well below the break-up despite the high accretion rate, Shu et al. (1994) developed a steady "X-wind model" which emanates from the point where the disk is corotating with its central star. Through the magnetic interaction between the central star and the inner edge of the disk where they assumed to be the corotation point, excess angular momentum brought in through the disk are extracted by X-winds. Subsequently, Ostriker \& Shu (1995) analyzed a steady state configuration of the magnetosphere between the central star and the disk and discussed how dipole fields become open and generate the "helmet streamer" by magnetic reconnection.

In the steady X-wind model, Shu et al. (1994) assumed that the dipole magnetic fields of the central star do not thread the disk except the inner edge where they assumed an enhanced magnetic diffusivity. It is possible, however, that the magnetic fields of the central star thread the disk either by enhanced turbulent magnetic diffusivity inside the disk or by the recoupling of the magnetic field and the disk matter due to the enhanced ionization rate near the central star.

In this Letter, we present the results of nonlinear, time-dependent MHD simulations of the interaction between the dipole magnetic field of the central star and the rotating disk threaded by the dipole field. 


\section{NUMERICAL MODELS}

We solve the resistive MHD equations in cylindrical coordinate $(r, \phi, z)$ by applying a modified Lax-Wendroff scheme with artificial viscosity (see Shibata 1983 and references therein). Axial symmetry is assumed. The effects of viscosity, radiative cooling and rotation of the central star are neglected. We take the z-axis parallel to the rotation axis of the disk and assume that the magnetic dipole moment is aligned with the rotational axis.

Exact solutions of the disk in rotational equilibrium are obtained under the simplifying assumptions for the distributions of angular momentum $L=L_{0} r^{a}$ and pressure $P=K \rho^{1+1 / n}$ where we adopted $a=0.494$ and $n=3$. The equation of force balance is integrated to give

$$
-\frac{G M}{\left(r^{2}+z^{2}\right)^{1 / 2}}+\frac{1}{2(1-a)} L_{0}^{2} r^{2 a-2}+(n+1) \frac{P}{\rho}=-\frac{G M}{r_{0}}+\frac{1}{2(1-a)} L_{0}^{2} r_{0}^{2 a-2}+(n+1) \frac{P_{0}}{\rho_{0}}
$$

where $M$ is the mass of the central star and the subscript 0 denotes quantities at the pressure maximum $\left(r_{0}, 0,0\right)$. The mass distribution inside the disk is determined by specifying the parameters $a, n$ and $P_{0} / \rho_{0}$. We use the normalizations $r_{0}=v_{K 0}=\rho_{0}=1$ where $v_{K 0}=\left(G M / r_{0}\right)^{1 / 2}$ is the Keplerian rotation speed at $r=r_{0}$. The unit of time is $\tau_{0}=r_{0} / v_{K 0}$. The mass distribution outside the disk is assumed to be that of the spherical isothermal corona surrounding the central star. The corona is assumed to be static at $t=0$.

The coronal density at $\left(0,0, r_{0}\right)$ is $\rho_{h}=2 \times 10^{-5} \rho_{0}$. The coronal temperature $T_{h}$ is $T_{h}=1.667$ $\times 10^{2} T_{0}$, where $T_{0}$ is the disk temperature at pressure maximum. The total simulation region in the $r-z$ plane is taken to be $0 \leq r \leq 13.1 r_{0}$ and $0 \leq z \leq 11.0 r_{0}$. The number of the mesh points used in our calculation is $\left(N_{r}, N_{z}\right)=(305,364)$. The grid spacings increase with $r$ and $z$.

We use free boundary conditions at the outer boundaries. At the equatorial plane $(z=0)$, we assume a symmetric boundary condition. We introduce a thin shell-like damping region (stellar surface) between $r_{i n}=\left(r^{2}+z^{2}\right)^{1 / 2}=0.336 r_{0}$ and $r_{s}=0.528 r_{0}$ 
where all quantities except magnetic fields are damped to the initial values. Alfvén waves can be reflected in this layer. All quantities are set to the initial values in stellar interior $\left(r \leq r_{i n}\right)$. Meanwhile magnetic fields are stress free at the outer boundaries.

As for resistivity, we assume anomalous resistivity $\eta(r, z)=\eta_{0}\left(v_{d} / v_{c}-1.0\right)^{2}$ when $v_{d}=|\mathbf{J}| / \rho$ exceeds the critical value $v_{c}$ where $\mathbf{J}$ is the current density. When $v_{d}<v_{c}, \eta(r, z)$ is set zero. This type of anomalous resistivity has been used in simulations of magnetic reconnection between the emerging flux and the overlying magnetic fields in the solar corona (see e.g., Shibata, Nozawa \& Matsumoto 1992, Yokoyama \& Shibata 1995).

\section{NUMERICAL RESULTS}

The results of the simulation of a typical model are shown in Figure 1 and Figure

2. The model parameters are $A_{1}=v_{s 0}^{2} /\left(\gamma v_{K 0}^{2}\right)=2.0 \times 10^{-3}, A_{2}=v_{A 0}^{2} / v_{K 0}^{2}=2.0 \times 10^{-4}$ where $v_{s 0}$ and $v_{A 0}$ are the sound speed and the Alfvén speed at $\left(r_{0}, 0,0\right)$, respectively. The adiabatic index $\gamma$ is $5 / 3$. We use the anomalous resistivity with $v_{c}=1000$ and $\eta_{0}=0.01$ in non-dimensional units. The plasma $\beta$ in the disk at the pressure maximum is $\beta_{0}=8 \pi P_{0} / B_{0}^{2}=2 A_{1} / A_{2}=20$.

Solid curves in Figure 1 show the magnetic field lines projected onto the $r-z$ plane. The color scale shows the temperature. The arrows show velocity vectors in the $r-z$ plane. The magnetic field lines connecting the central star and the disk are twisted by the rotation of the disk. As the magnetic twist accumulates, the magnetic loops begin to inflate. They expand quasi-statically in the early stage but later $\left(t>2 \tau_{0}\right)$, they expand dynamically. A current sheet is formed inside the expanding loops and the current density exceeds the threshold for the onset of anomalous resistivity. Around $t=2.4 \tau_{0}$, magnetic reconnection takes place in the current sheet. Outgoing magnetic island and post flare loops 
are formed after the reconnection $\left(t=2.68 \tau_{0}\right)$. Hot plasmas created by the Joule heating and compressional heating are confined in the outgoing magnetic island. The speed of outflowing hot plasma is the order of the local Alfvén speed $v_{A} \sim(2-5) v_{K 0}$. Crest-shaped fast MHD shock waves are formed in front of the expanding magnetic loops. By parametric survey, we confirmed that when the coronal density is lower the speed of reconnection flow and temperature are higher because local Alfvén speed increases.

Inside the magnetic island, magnetic field lines are almost toroidal. Since they are detached from the star and the disk, the mass and the total angular momentum of the rotating toroid is conserved. By equating the centrifugal force $l^{2} / r^{3}$ where $l$ is the specific angular momentum with the radial component of gravity $G M r /\left(r^{2}+z^{2}\right)^{3 / 2}$, we get $r / z \rightarrow 0$ as $z \rightarrow \infty$; the plasmoid is collimated toward the rotational axis. The azimuthal magnetic fields also helps collimating the outflow by self-pinching effect (see Uchida \& Shibata 1985; Shibata \& Uchida 1986). Although the computational domain in our simulation is too small to show the collimation, the velocity vectors at $t=4.0 \tau_{0}$ show a tendency to point toward the polar directions.

The magnetic field lines near the disk have small angle with respect to the equatorial plane. This configuration is favorable for the appearance of magneto-centrifugal winds from accretion disks (e.g., Blandford \& Payne 1982). The speed of the disk wind is the order of the Keplerian rotation speed at the footpoint of the field lines on the disk. Since the density is high, the mass outflow rate of the cold disk wind is several times larger than that of the hot plasma jet driven by the reconnection.

Figure 2 shows the density distribution (gray scale) and isocontours of magnetic energy density. Just after the reconnection, the density of the reconnection region decreases due to the heating and expansion. During the later evolution, the density increases to $\rho \sim 10^{-5} \rho_{0}$, because the colder gas that flows out of the disk refills the region. This justifies rather high 
initial coronal density $\left(\rho_{h} \sim 2 \times 10^{-5} \rho_{0}\right)$ in our model.

Figure 3 shows the time history of various energies. The solid curve in the lower panel shows the total magnetic energy $E_{m a g}$ in the simulation region. The dotted curve shows the magnetic energy $E_{h, m a g}$ of hot plasma $\left(T>1.2 T_{h}\right)$. The magnetic energy increases almost linearly with time until magnetic reconnection takes place. The kink around $t=2.4 \tau_{0}$ is due to the magnetic reconnection. The magnetic energy of hot component saturates and slightly decreases. In the MHD simulations of solar coronal magnetic loops which subject to the helicity injection, Kusano, Suzuki \& Nishikawa (1995) noted similar kink and explained it by extending the Woltjer \& Taylor's minimum energy principle (Woltjer 1958, Taylor 1974).

The solid curve and the dotted curve in the top panel of Figure 3 show thermal energy $E_{h, t h}$, and the kinetic energy $E_{h, k i n}$ of hot plasma $\left(T>1.2 T_{h}\right)$, respectively. Rotational energy is included in $E_{h, k i n}$. The kinetic energy slowly increases during quasi-hydrostatic evolution $\left(t<2 \tau_{0}\right)$. The kinetic energy increases more steeply when the equilibrium is lost $\left(t \sim 2.0 \tau_{0}\right)$. Since the magnetic loops expand rapidly, the rate of increase in the coronal thermal energy $E_{h, t h}$ decreases during $t=2.0-2.4 \tau_{0}$. The coronal thermal energy increases more steeply after magnetic reconnection at $t=2.4 \tau_{0}$. These curves clearly show the bifurcation of the system into a different energy state after magnetic reconnection.

It should be noted that the energy injected into the corona through the rotation of the disk $\left(E_{i n j}=E_{m a g}+E_{h, t h}+E_{h, k i n}\right)$ increases almost linearly with time even after magnetic reconnection because magnetic helicity is continuously injected from the disk. At $t=3.0 \tau_{0}$, about $70 \%$ of the injected energy goes into the magnetic energy, $18 \%$ to the thermal energy, and $12 \%$ to the kinetic energy. The thermal energy of hot plasma at $t=3.0 \tau_{0}$ is about $40 \%$ of the initial total magnetic energy ( $15 \%$ of the total magnetic energy at $t=3 \tau_{0}$ ). 


\section{DISCUSSION}

In this Letter, we demonstrated that if the dipole magnetic field of the central star is connected to the rotating disk which is not co-rotating with the central star, the dipole magnetic fields can disrupt by imposed twists within one rotation of the disk. We schematically summarize our numerical results in Figure 4. Our 2.5D nonlinear MHD simulation confirmed the previous expectation from the equilibrium calculations by Zylstra (1988) that magnetic reconnection will be triggered by the loss of equilibrium due to the helicity injection. We also showed that hot plasmoids are ejected in bipolar directions with velocity 2-5 times the Keplerian rotation speed around the inner edge of the disk. Plasma heating occurs both by the Joule heating in the current sheet developed inside the inflating magnetic loops and by shock waves created by magnetic reconnection. The plasma temperature can rise up to the temperature determined by $v_{s} \sim v_{A} \sim 2-5 v_{K}$. Since $v_{K} \sim 170 \mathrm{~km} \mathrm{~s}^{-1}$ at $r=0.03 \mathrm{AU}$ around $1 M_{\odot}$ star, the temperature of the plasmoid and the post flare loop can be $10^{7}-10^{8} \mathrm{~K}$. According to the observation of an X-ray flare from a protostar (Koyama et al. 1996b), the emission spectrum of protostellar flare extends up to $10 \mathrm{keV}$. This result is consistent with the above plasma temperature expected from magnetospheric flares in protostars.

Observation of X-ray flares by ASCA (Koyama et al. 1996b) showed that the e-folding time of the flare is $\sim 5 \times 10^{4} \mathrm{sec}$ and the total energy of the flare is $10^{36} \mathrm{erg}$. The size of X-ray emitting region derived from the emission measure $\left(\sim 10^{54} \mathrm{~cm}^{-3}\right)$ and flare decay time is the order of $10^{11} \mathrm{~cm}$. The number density of the flaring plasma is $n_{\text {flare }} \sim 10^{10.5}$ $\mathrm{cm}^{-3}$. This rather high density can be explained by the dynamical refillment of the corona by the mass outflow from the disk (see Figure 2). When the X-ray emitting plasmas are confined in loops, their size should be larger than the radius of the protostar. Our numerical results are consistent with this observational constraint because hot plasmas are created in 
gigantic loops several times larger than the stellar radius. The mass flux of the hot plasma outflow obtained from simulation is

$$
\dot{M} \sim 10^{-8}\left(\frac{r_{0}}{0.03 \mathrm{AU}}\right)^{2}\left(\frac{v_{K 0}}{170 \mathrm{~km} \mathrm{~s}^{-1}}\right)\left(\frac{n_{\text {flare }}}{3 \cdot 10^{10} \mathrm{~cm}^{-3}}\right) M_{\odot} \mathrm{yr}^{-1}
$$

After the X-ray emitting gas is cooled down (cooling time $\sim 1$ day), it will be observed as optical jets. The mass flux of the hot plasma is consistent with the observation of optical jets.

Next, let us discuss whether the magnetospheric flares can release the observed total energy of X-ray flares. In our simulation, initial magnetic energy is thermalized within one rotation period $\left(\sim 10^{5} \mathrm{sec}\right.$ at $\left.0.03 \mathrm{AU}\right)$. If the size of the flaring region is $d \sim 10^{11} \mathrm{~cm}$ and the mean strength of magnetic field in the flaring region is $B \sim 100-200 \mathrm{G}$, the released energy $L \sim\left(B^{2} / 8 \pi\right) d^{3} \sim 10^{36}$ erg is the order of the observed luminosity.

We checked the dependence of numerical results on resistivity. When the threshold for anomalous resistivity $v_{c}$ is larger, the plasma temperature and the speed of reconnection flow are larger because magnetic reconnection does not take place until large amount of magnetic energy is accumulated. Since the speed of the jet is larger, the magnetic island is deformed into a "heart shape" as shown in Figure 1. This feature has also been noted by Choe (1995) when he simulated the solar magnetic arcades under photospheric shearing motion. When the resistivity is uniform or $v_{c}$ is small, the magnetic island is more circular.

We would like to comment on the relation between our model and the $\mathrm{X}$-wind model by Shu et al. (1994). They considered that the magnetic fields of the central star can thread the disk only in the prescribed small diffusive transition zone near the point where the disk corotates with the central star. Interior to this radius, magnetic field lines are closed and connected to the surface of the star. Along the open magnetic field lines, X-winds emanate from the small diffusive zone around the inner edge of the disk. This configuration is similar to the final stage of our simulation in which the magnetic field lines after reconnection 
become partially open, and dense, cold winds flow out from the disk along the partially open field lines. Although we cannot continue the simulation until steady state is reached, we could show some essential components of the X-wind model. Of course, we need to include the effect of the stellar rotation in order to study the angular momentum exchange between the star and the disk. We want to extend our study in such direction in near future.

We thank Prof. K. Koyama for providing us the results of their hard X-ray observation by ASCA prior to publication. We would like to thank Dr. S. Miyaji, Dr. Masahiko Hayashi, Prof. T. Tajima, Dr. J.C.L. Wang, Dr. T. Kudoh and Dr. S. Hirose for their helpful discussions. Prof. F. Shu is acknowledged for introducing us the X-wind model during his visit to the National Astronomical Observatory, Japan. We also thank Prof. F. Lamb for pointing out the related work by Zylstra (1988). Numerical computations were carried out by using Fujitsu VPP500 at the Institute of Space and Astronautical Science and VPP500 at the Japan Atomic Energy Research Institute. This work is supported in part by the grant of Ministry of Education and Culture, Japan (07640348). 


\section{REFERENCES}

Blandford, R. D., \& Payne, D. G. 1982, MNRAS, 199, 883

Camenzind, M. 1990 , Reviews in Modern Astronomy, 3, p.234

Choe, G. S. 1995, PhD thesis, Univ. Alaska

Ghosh, P., \& Lamb, F. K. 1979, ApJ, 232, 259

Ghosh, P., \& Lamb, F. K. 1991, in Neutron stars : Theory and observation, ed. Ventura, J. \& Pines, D., (Dortrecht: Kluwer), p. 363

Hirose, S. 1994, PhD thesis, Univ. Tokyo

Koyama, K., Maeda, Y., Ozaki, M., Ueno, S., Kamata, Y., Tawara, Y., Skinner, S., \& Yamauchi, S. 1994, PASJ, 46, L125

Koyama K. 1996a, IAU Colloquim No. 153, Magnetodynamic Phenomena in the Solar Atmosphere, ed. Kosugi \& Uchida, (Dortrecht: Kluwer) in press

Koyama, K., Ueno, S., Kobayashi, N., Feigelson, E. D. 1996b, preprint

Kusano, K., Suzuki, Y., \& Nishikawa, K. 1995, ApJ, 441, 942

Linker, J. A., \& Mikić, Z. 1995, ApJ, 438, L45

Lovelace, R.V.E., Romanova, M.M., \& Bisnovatyi-Kogan, G.S. 1995, MNRAS, 275, 244

Lynden-Bell, D., \& Boily, C. 1994, MNRAS, 267, 146

Mikić, Z., \& Linker, J. A. 1994, ApJ, 430, 898

Ostriker, E. C. \& Shu, H. F. 1995, ApJ, 447, 813

Shibata, K. 1983, PASJ, 35, 263 
Shibata, K., \& Uchida, Y. 1986, PASJ, 38, 631

Shibata, K., Nozawa, S., \& Matsumoto, R. 1992, PASJ, 44, 265

Shu, F., Najita, J., Ostriker, Eve., Wilkin, F., Ruden, S. \& Lizano, S. 1994, ApJ, 429, 781

Taylor, J. B. 1974, Phys. Rev. Lett., 33, 1139

Uchida, Y., \& Shibata, K. 1985, PASJ, 37, 515

Woltjer, L. 1958, Proc. Nat. Acad. Sci. USA, 44, 489

Yokoyama, T., \& Shibata, K. 1995, Nature, 375, 42

Zylstra, G. J. 1988, PhD thesis, Univ. Illinois 
Fig. 1. - The result of the numerical simulation for a model with $A_{1}=v_{s 0}^{2} /\left(\gamma v_{K 0}^{2}\right)=$ $2.0 \times 10^{-3}, A_{2}=v_{A 0}^{2} / v_{K 0}^{2}=2.0 \times 10^{-4}$ and $\rho_{h} / \rho_{0}=2.0 \times 10^{-5}$. The color scale shows the temperature normalized by $T_{k}=\mu v_{K 0}^{2} / R$, here $\mu$ is mean molecular weight and $R$ is gas constant. The initial coronal temperature is $T_{h} / T_{k}=1 / 3$. Solid curves denote magnetic field lines. The region of closely spaced field lines is included to identify the reconnection region in the subsequent frames. Arrows depict velocity vectors in $r-z$ plane. The unit velocity is shown by arrows at the top right of each panel.

Fig. 2.- Time variation of the density distribution. Color scale shows the density. Solid curves denote isocontours of magnetic energy density. Arrows depict the velocity vector.

Fig. 3.- Time history of various energies normalized by the initial magnetic energy. Upper panal shows the thermal energy $E_{h, t h}$ (solid curve) and kinetic energy $E_{h, k i n}$ (dotted curve) of hot component,

$$
E_{h, t h}=2 \pi \int_{0}^{z_{\max }} \int_{r_{s}}^{r_{\max }}[P /(\gamma-1)] r d r d z, \quad E_{h, k i n}=2 \pi \int_{0}^{z_{\max }} \int_{r_{s}}^{r_{\max }}\left(\rho v^{2} / 2\right) r d r d z
$$

The lower panel shows the total magnetic energy $E_{m a g}$ (solid curve) and that of the hot component $E_{h, m a g}$ (dotted curve).

Fig. 4.- A schematic picture of numerical results. Hot plasma jet ejected from the flaring region corresponds to the optical jet. Cold, dense wind emanating from the disk may explain high velocity neutral winds. 

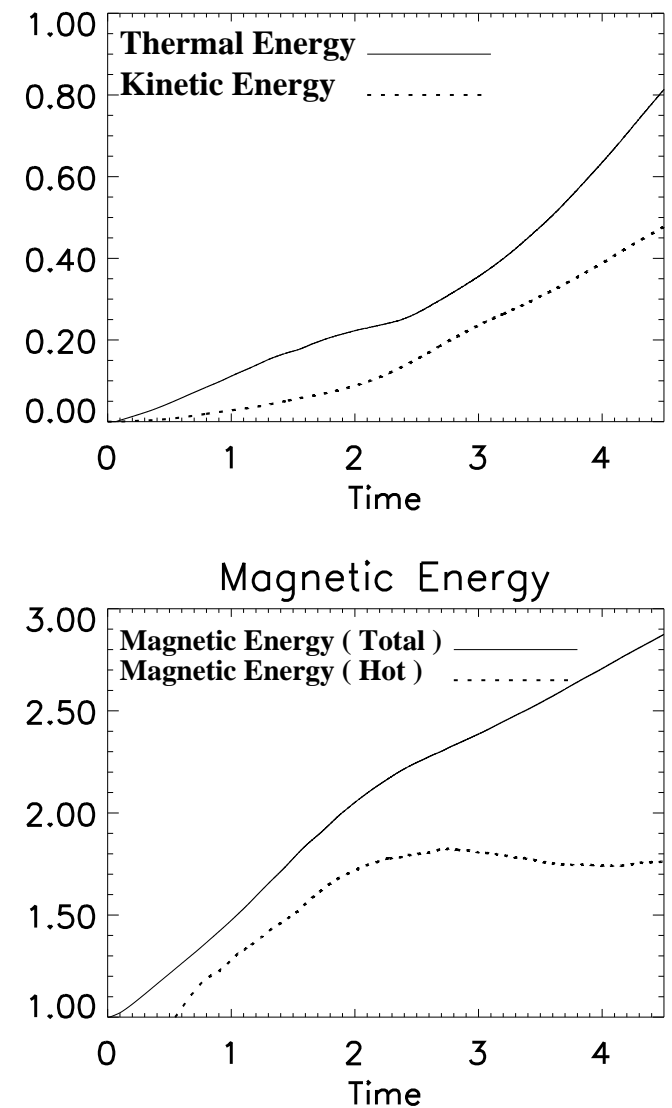
fast shock



